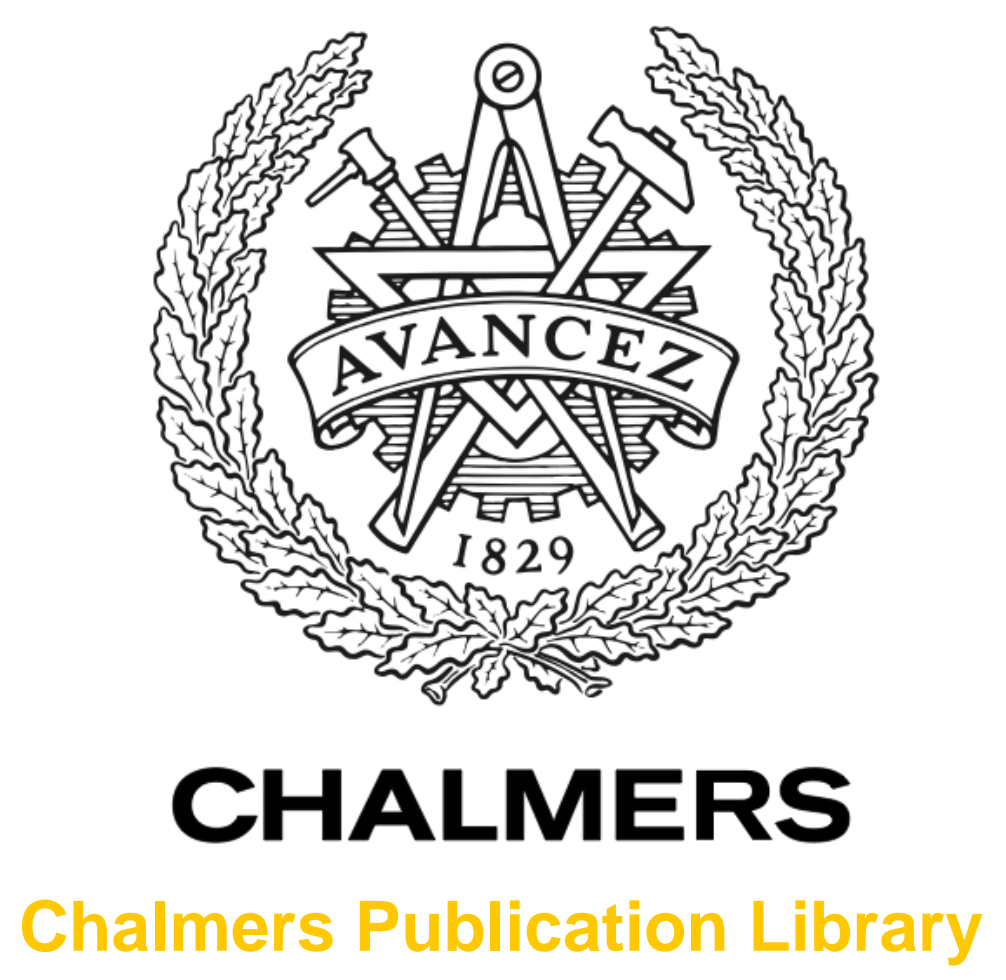

Spatially Coupled LDPC Codes for Two-User Decode-and-Forward Relaying'

This document has been downloaded from Chalmers Publication Library (CPL). It is the author's version of a work that was accepted for publication in:

2012 7th International Symposium on Turbo Codes and Iterative Information Processing, ISTC 2012, Gothenburg, 27 - 31 August 2012 (ISSN: 2165-4700)

Citation for the published paper:

Schwandter, S. ; Graell i Amat, A. ; Matz, G. (2012) "Spatially Coupled LDPC Codes for Two-User Decode-and-Forward Relaying'". 2012 7th International Symposium on Turbo Codes and Iterative Information Processing, ISTC 2012, Gothenburg, 27 - 31 August 2012 pp. 46-50.

http://dx.doi.org/10.1109/ISTC.2012.6325196

Downloaded from: http://publications.lib.chalmers.se/publication/167859

Notice: Changes introduced as a result of publishing processes such as copy-editing and formatting may not be reflected in this document. For a definitive version of this work, please refer to the published source. Please note that access to the published version might require a subscription.

Chalmers Publication Library (CPL) offers the possibility of retrieving research publications produced at Chalmers University of Technology. It covers all types of publications: articles, dissertations, licentiate theses, masters theses, conference papers, reports etc. Since 2006 it is the official tool for Chalmers official publication statistics. To ensure that Chalmers research results are disseminated as widely as possible, an Open Access Policy has been adopted.

The CPL service is administrated and maintained by Chalmers Library. 


\title{
Spatially Coupled LDPC Codes for Two-User Decode-and-Forward Relaying
}

\author{
Stefan Schwandter ${ }^{\dagger}$, Alexandre Graell i Amat ${ }^{\S}$, and Gerald Matz ${ }^{\dagger}$ \\ ${ }^{\dagger}$ Institute of Telecommunications, Vienna University of Technology, Gusshausstrasse 25/389, A-1040 Vienna, Austria \\ $\S$ Department of Signals and Systems, Chalmers University of Technology, SE-412 96 Gothenburg, Sweden \\ Email: \{sschwand,gmatz\}@nt.tuwien.ac.at, alexandre.graell@chalmers.se
}

\begin{abstract}
We present a decode-and-forward transmission scheme that is based on spatially coupled LDPC codes and applies to a network consisting of two sources, one relay, and one destination. The relay performs network coding to achieve full diversity. We prove analytically that the proposed scheme achieves the Shannon limit on the binary erasure relay channel for symmetric channel conditions. Using density evolution, we furthermore demonstrate that our scheme approaches capacity also for asymmetric channel conditions.
\end{abstract}

\section{INTRODUCTION}

The three-node relay channel was introduced by van der Meulen in [1] and the first capacity results were presented in [2]. While the capacity for the general relay channel is still unknown, recent years have seen a vast amount of research on the topic, both in the information theory and coding communities. The decode-and-forward (DF) relaying scheme was introduced in [2] and achieves the capacity of the relay channel for certain special cases. With DF, the relay decodes the source data and provides a re-encoded copy of the source message to the destination. Several papers have considered practical implementations of DF based on convolutional codes [3], capacity-approaching turbo codes [4] or low-density parity check (LDPC) codes [5]. In [6], so-called bilayer LDPC codes were introduced and were shown to closely approach the theoretical DF rate.

Recently, it has been proved that regular spatially coupled (SC) LDPC codes achieve capacity on the binary erasure channel (BEC) [7]. For this reason, SC-LDPC codes are excellent candidates for the design of relay schemes whose performance is close to the information-theoretic limits. In [8] it was shown that bilayer SC-LDPC codes can actually achieve the Shannon limit of a DF relay system with orthogonal BEC links. Since the SC-LDPC code ensembles are regular, the design complexity is very low compared to schemes based on irregular LDPC code ensembles, which require extensive optimization.

In more realistic networks, one relay is shared by multiple users. The information-theoretic model for this scenario is the multiple-access relay channel (MARC), first studied in [9]

The work of S. Schwandter and G. Matz was funded by FWF Grant S10606 and WWTF Grant ICT08-44. A. Graell i Amat was supported by the Swedish Agency for Innovation Systems (VINNOVA) under the P36604-1 MAGIC project.

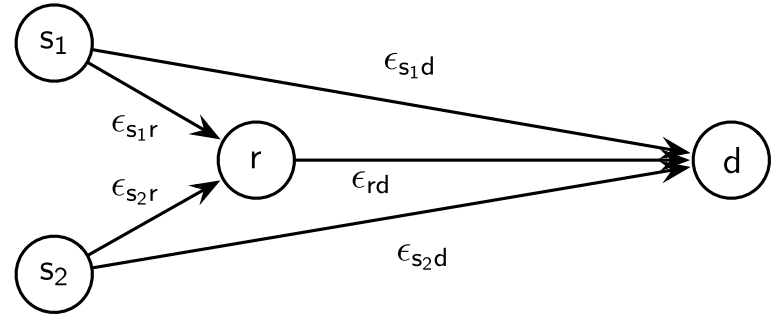

Fig. 1. Two-user relay network in which all links are modeled as binary erasure channels (links are labeled with erasure probabilities).

and later specialized to time-division multiple-access (TDMA) channels in [10]. While the achievable rate of the TDMAMARC is the same as that of two separate relay channels there is a potential diversity gain. To exploit that gain, the relay has to combine the information of the sources via network coding [11]. Network coding can be achieved implicitly by an appropriate design of the channel codes, a technique termed joint network-channel coding. For the TDMA-MARC, several coding schemes have been proposed based on regular LDPC codes [12], irregular LDPC codes [13], and seriallyconcatenated codes [14].

In this paper, we design bilayer SC-LDPC codes for a halfduplex DF relaying system with two sources, one relay, and one destination, thereby extending [8]. The code construction implicitly provides network coding at the relay node to achieve the maximum diversity gain. For the BEC and the case of symmetric channel conditions, we prove analytically that the proposed scheme achieves the Shannon limit. For asymmetric channel conditions, we provide density evolution (DE) thresholds for our scheme that show a performance close to the Shannon limit and suggest that capacity is also achieved in this case.

\section{SYSTEM MODEL}

We consider the relay network depicted in Fig. 1. There are four nodes, sources one and two $\left(\mathrm{s}_{1}, \mathrm{~s}_{2}\right)$, relay $(\mathrm{r})$ and destination (d). One transmission block is split into three phases in order to orthogonalize the transmission links; in each phase one node transmits and the others listen. In phase $i$ ( $i=1,2$ for the rest of the paper), source $\mathrm{s}_{i}$ transmits a length- $n_{i}$ codeword $\mathbf{x}_{i}$, obtained by encoding $k$ information bits with a channel code of rate $R_{i}=k / n_{i}$. The relay 
receives $\mathbf{x}_{1}$ and $\mathbf{x}_{2}$ over two BECs with erasure probabilities (EPs) $\epsilon_{\mathrm{s}_{i} \mathrm{r}}$. The destination receives the same codewords over BECs with EPs $\epsilon_{\mathrm{S}_{i} \mathrm{~d}}$. The relay decodes both transmissions, generates $k_{\mathrm{r}}$ additional parity bits and encodes them into a codeword of length $n_{\mathrm{r}}$ using a channel code of rate $R_{\mathrm{r}}$. In the third time slot, the joint network-channel-coded information is forwarded to the destination over a BEC with EP $\epsilon_{\mathrm{rd}}$. The whole transmission block consists of $N=n_{1}+n_{2}+n_{\mathrm{r}}$ bits. We define the duty cycle of each phase as $\theta_{j}=n_{j} / N$, $j=\{1,2, \mathrm{r}\}$. Since we assume, without loss of generality, that each source transmits the same number of information bits, the effective rate $R=k / N$ is equal for both sources. The sum rate of the system is $R_{\text {sum }}=2 k / N=2 R$.

\section{THEORETICAL LIMITS}

The information-theoretic limits of the TDMA-MARC with DF relaying have been presented in [10, p. 95]. Denote by $C=$ $1-\epsilon$ the capacity of a BEC. Under the conditions $C_{\mathrm{s}_{i} \mathrm{r}} \geq C_{\mathrm{s}_{i} \mathrm{~d}}$ and $C_{\mathrm{rd}} \geq C_{\mathrm{s}_{i} \mathrm{~d}}$, the optimal allocation of transmission time between the three phases is

$$
\begin{aligned}
& \theta_{1}^{*}=\frac{C_{\mathrm{rd}}}{(1+\kappa) C_{\mathrm{rd}}+2 C_{\mathrm{s}_{1} \mathrm{r}}-C_{\mathrm{s}_{1} \mathrm{~d}}-\kappa C_{\mathrm{s}_{2} \mathrm{~d}}}, \\
& \theta_{2}^{*}=\kappa \theta_{1}^{*}, \quad \theta_{\mathrm{r}}^{*}=1-\theta_{1}^{*}-\theta_{2}^{*},
\end{aligned}
$$

where $\kappa=\frac{C_{\mathrm{s}_{1}^{\mathrm{r}}}}{C_{\mathrm{s}_{2} \mathrm{r}}}$. The achievable rate per user is

$$
R_{\max }=\frac{C_{\mathrm{s}_{1} \mathrm{r}} C_{\mathrm{rd}}}{(1+\kappa) C_{\mathrm{rd}}+2 C_{\mathrm{s}_{1} \mathrm{r}}-C_{\mathrm{s}_{1} \mathrm{~d}}-\kappa C_{\mathrm{s}_{2} \mathrm{~d}}} .
$$

To achieve $R_{\max }$, capacity-achieving codes on both sourcerelay links are used in the first two transmission phases, such that the relay can decode successfully. In the third phase, the relay transmits additional parity bits based on the source data to the destination. The destination effectively sees two new overall codes with lower rates, which are capacity achieving for the source-destination links, and decodes them based on the channel observations of all three transmission phases.

\section{BILAYER SC-LDPC CODES}

\section{A. SC-LDPC codes}

We briefly introduce SC-LDPC codes. For a detailed description of the ensemble used in this paper see [7].

A regular $(l, r)$ SC-LDPC code with variable node degree $l$ and check node degree $r$ is defined by an infinite parity check matrix

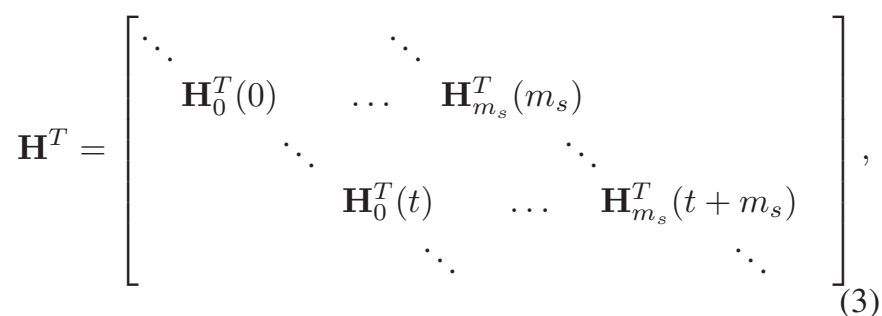

where superscript $T$ denotes the transpose. The Tanner graph of such a code is divided into "positions" or "time instants" $t$, similar to the code frames in classical convolutional codes. At each position $t \in(-\infty, \infty)$ there are $M$ variable nodes, and $M \frac{l}{r}$ check nodes. This is reflected in the parity check matrix by the fact that each submatrix $\mathbf{H}_{j}^{T}(t+j), j \in\left[0, m_{s}\right]$, is a sparse $M \times\left(M \frac{l}{r}\right)$ binary matrix. For our application we will consider terminated spatially coupled code ensembles, where we assume that the codeword is restricted to $t \in[1, L]$.

In this paper, we use the $(l, r, L, w, M)$ ensemble described in [7], where each of the $l$ edges of a variable node at position $t$ is randomly connected to a check node in the range $[t, t+$ $w-1]$ using an i.i.d. uniform distribution $\left(w=m_{s}+1\right)$. The randomization results in simple DE equations and thus renders the ensemble accessible to analysis. For transmission over the $\mathrm{BEC}$, the code rate of the $(l, r, L, w, M)$ ensemble tends to the one of the underlying block code ensemble (whenever we omit $M$ in the rest of the paper, we assume $M \rightarrow \infty$ )

$$
\lim _{w \rightarrow \infty} \lim _{L \rightarrow \infty} R(l, r, L, w)=1-\frac{l}{r} .
$$

Furthermore, its belief propagation (BP) threshold $\epsilon^{\mathrm{BP}}$ tends to the MAP threshold $\epsilon^{\mathrm{MAP}}$ of the underlying ensemble,

$$
\lim _{L, w \rightarrow \infty} \epsilon^{\mathrm{BP}}(l, r, L, w)=\lim _{L, w \rightarrow \infty} \epsilon^{\mathrm{MAP}}(l, r, L, w)=\epsilon^{\mathrm{MAP}}(l, r),
$$

when letting $L$ and $w$ go to infinity (in that order). On the BEC, the Shannon limit for transmission at rate $R$ is given by the $\mathrm{EP} \epsilon^{\mathrm{Sh}}=1-R$ below which reliable (error-free) transmission is possible. The MAP threshold of a regular LDPC block code ensemble tends to the Shannon limit exponentially fast in $l$ if the design rate $R(l, r)$ is kept fixed (cf. [7, Lemma 8]):

$$
\lim _{l \rightarrow \infty} \epsilon^{\mathrm{MAP}}\left(l, r=\frac{l}{1-R(l, r)}\right)=1-R=\epsilon^{\mathrm{Sh}} .
$$

\section{B. Two-user bilayer SC-LDPC code}

The sources use codes from the ensembles $\mathcal{C}_{1}\left(l_{1}, r_{1}, L_{1}, w_{1}, M_{1}\right)$ and $\mathcal{C}_{2}\left(l_{2}, r_{2}, L_{2}, w_{2}, M_{2}\right)$, respectively, with design rates $R_{i} \triangleq \frac{k}{n_{i}}=\frac{k}{L M_{i}}=C_{\mathrm{s}_{i} \mathrm{r}}$, and parity check matrices $\mathbf{H}^{1}$ and $\mathbf{H}^{2}$. They comprise the first layer of the bilayer structure. Assuming perfect transmissions on the $s_{i}$-r links, the relay recovers the codewords $\mathbf{x}_{1}$ and $\mathbf{x}_{2}$ transmitted by the sources. It generates $k_{\mathrm{r}}$ additional syndrome bits according to

$$
\mathbf{s}=\left[\begin{array}{ll}
\mathbf{H}_{\text {synd }}^{1} & \mathbf{H}_{\text {synd }}^{2}
\end{array}\right]\left[\begin{array}{l}
\mathbf{x}_{1} \\
\mathbf{x}_{2}
\end{array}\right],
$$

using parity check matrices from the code ensembles $\mathcal{C}_{\text {synd }}^{1}\left(l_{\text {synd }}^{1}, r_{\text {synd }}^{1}, L_{1}, w_{1}, M_{1}\right) \quad$ and $\mathcal{C}_{\text {synd }}^{2}\left(l_{\text {synd }}^{2}, r_{\text {synd }}^{2}, L_{2}, w_{2}, M_{2}\right)$. These codes constitute the second layer of the bilayer code.

The syndrome bits are transmitted to the destination protected by a code of rate $R_{\mathrm{r}}=C_{\mathrm{rd}}$. Assuming that the destination can recover the syndrome bits perfectly, it can now decode the source bits using the parity check matrix $\mathbf{H}$ of the overall code according to

$$
\mathbf{H}\left[\begin{array}{l}
\mathbf{x}_{1} \\
\mathbf{x}_{2}
\end{array}\right]=\left[\begin{array}{cc}
\mathbf{H}^{1} & \mathbf{0} \\
\mathbf{0} & \mathbf{H}^{2} \\
\mathbf{H}_{\text {synd }}^{1} & \mathbf{H}_{\text {synd }}^{2}
\end{array}\right]\left[\begin{array}{l}
\mathbf{x}_{1} \\
\mathbf{x}_{2}
\end{array}\right]=\left[\begin{array}{l}
\mathbf{0} \\
\mathbf{0} \\
\mathbf{s}
\end{array}\right] .
$$


For simplicity, we assume $L_{1}=L_{2}$ and $w_{1}=w_{2}$ without loss of generality. This means that to achieve different block sizes for $s_{1}$ and $s_{2}$, we choose a different number of variables per time instant for each user, $M_{1} \neq M_{2}$. We note that the overall matrix $\mathbf{H}$ does not have the band structure of an SC-LDPC code (cf. (3)); rather, it consists of four concatenated band matrices. Nevertheless, we will analytically prove capacityachieving performance for symmetric channel conditions below and we conjecture that capacity is achieved under general channel conditions.

From (5) we see that the syndrome bits in general depend on code bits from both sources. Of the total number $k_{\mathrm{r}}$ of syndrome bits, effectively $k_{\mathrm{r}}^{1}$ and $k_{\mathrm{r}}^{2}$ bits are used to respectively decode $\mathrm{s}_{1}$ and $\mathrm{s}_{2}$ at the destination $\left(k_{\mathrm{r}}=k_{\mathrm{r}}^{1}+k_{\mathrm{r}}^{2}\right)$. The number of bits from source $\mathrm{s}_{i}$ involved in one of the $k_{\mathrm{r}}$ checks is given by the check node degree $r_{\text {synd }}^{i}$. The effective number of syndrome bits for source $\mathrm{s}_{i}$ is therefore

$$
k_{\mathrm{r}}^{i}=k_{\mathrm{r}} \mu_{i}, \quad \text { with } \mu_{i} \triangleq \frac{r_{\text {synd }}^{i}}{r_{\text {synd }}^{1}+r_{\text {synd }}^{2}} .
$$

The design rules for bilayer LDPC codes require

$$
k_{r}^{i}=n_{i}\left(C_{\mathrm{s}_{i} \mathrm{r}}-C_{\mathrm{s}_{i} \mathrm{~d}}\right) .
$$

This choice results in rates of the bilayer codes that are equal to the capacities of the $\mathrm{s}_{i}$-d channels,

$$
R_{\mathrm{bl}}^{i} \triangleq \frac{n_{i}-\left(n_{i}-k_{i}+k_{\mathrm{r}}^{i}\right)}{n_{i}}=\frac{k_{i}-k_{\mathrm{r}}^{i}}{n_{i}}=C_{\mathrm{s}_{i} \mathrm{~d}} .
$$

The destination will therefore be able to successfully decode both users' messages if capacity-achieving codes are used.

The design rates for $\mathcal{C}_{\text {synd }}^{i}\left(l_{\text {synd }}^{i}, r_{\text {synd }}^{i}, L, w, M_{i}\right)$ follow from (7) and the optimum time allocation (1), $\frac{\theta_{2}^{*}}{\theta_{1}^{*}}=\frac{n_{2}^{*}}{n_{1}^{*}}=\frac{C_{\mathrm{s}_{1} \mathrm{r}}}{C_{\mathrm{s}_{2} \mathrm{r}}}$ :

$$
\begin{aligned}
& R_{\text {synd }}^{1} \triangleq 1-\frac{k_{\mathrm{r}}}{n_{1}}=1-\left(C_{\mathrm{s}_{1} \mathrm{r}}\left(2-\frac{C_{\mathrm{s}_{2} \mathrm{~d}}}{C_{\mathrm{s}_{2} \mathrm{r}}}\right)-C_{\mathrm{s}_{1} \mathrm{~d}}\right), \\
& R_{\text {synd }}^{2} \triangleq 1-\frac{k_{\mathrm{r}}}{n_{2}}=1-\left(C_{\mathrm{s}_{2} \mathrm{r}}\left(2-\frac{C_{\mathrm{s}_{1} \mathrm{~d}}}{C_{\mathrm{s}_{1} \mathrm{r}}}\right)-C_{\mathrm{s}_{2} \mathrm{~d}}\right) .
\end{aligned}
$$

Combining (6) and (7) gives the design goal for the ratio of $r_{\text {synd }}^{1}$ and $r_{\text {synd }}^{2}$,

$$
\mu \triangleq \frac{r_{\text {synd }}^{1}}{r_{\text {synd }}^{2}}=\frac{k_{\mathrm{r}}^{1}}{k_{\mathrm{r}}^{2}}=\frac{C_{\mathrm{s}_{2} \mathrm{r}}\left(C_{\mathrm{s}_{1} \mathrm{r}}-C_{\mathrm{s}_{1} \mathrm{~d}}\right)}{C_{\mathrm{s}_{1} \mathrm{r}}\left(C_{\mathrm{s}_{2} \mathrm{r}}-C_{\mathrm{s}_{2} \mathrm{~d}}\right)} .
$$

Finally, combining (8)-(11) allows to obtain the resulting bilayer code rates when $R_{1}, R_{2}, R_{\text {synd }}^{1}, R_{\text {synd }}^{2}$ and $\mu$ are given:

$$
R_{\mathrm{bl}}^{1}=R_{1}-\frac{1-R_{\mathrm{synd}}^{1}}{1+\mu^{-1}}, \quad R_{\mathrm{bl}}^{2}=R_{2}-\frac{1-R_{\mathrm{synd}}^{2}}{1+\mu} .
$$

\section{DEnSity EVOLUtion ANALYSis}

In the following we show that for symmetric channel conditions, defined as $\epsilon_{\mathrm{s}_{1} \mathrm{r}}=\epsilon_{\mathrm{s}_{2} \mathrm{r}}=\epsilon_{\mathrm{sr}}, \epsilon_{\mathrm{s}_{1} \mathrm{~d}}=\epsilon_{\mathrm{S}_{2} \mathrm{~d}}=$ $\epsilon_{\mathrm{sd}}$, the proposed scheme achieves the highest possible DF rate on the TDMA-MARC with BEC links. We call the two-user bilayer ensemble consisting of $\mathcal{C}_{i}(l, r, L, w)$ and $\mathcal{C}_{\text {synd }}^{i}\left(l_{\text {synd }}, r_{\text {synd }}, L, w\right)$ the $\mathcal{C}_{\mathrm{bl}}\left(l, l_{\text {synd }}, r, r_{\text {synd }}, L, w\right)$ ensemble.
Lemma 1. For the case of symmetric channel conditions and $r_{\text {synd }}=r / 2$, the two-user bilayer code $\mathcal{C}_{b l}\left(l, l_{\text {synd }}, r, r / 2, L, w\right)$ achieves the same DE threshold for each source-destination link as the single-layer code $\mathcal{C}\left(l+l_{\text {synd }}, r, L, w\right)$.

Proof: First note that the choice $r_{\text {synd }}=r / 2$ allows us to write the DE equations in a form that we need to proof the capacity-achieving property. However, this does not restrict the possible rates available in the system and therefore the result is general. For source $\mathrm{s}_{i}$, we denote the messages (erasure probabilities) sent from a variable node at position $t$ in iteration $I$ to a check node in the first and the second layer as $p_{i}^{(t, I)}$ and $p_{\text {synd }, i}^{(t, I)}$, respectively. The messages from check nodes at position $t$ in iteration $I$ to variable nodes are called $q_{i}^{(t, I)}$ and $q_{\text {synd }, i}^{(t, I)}$. For $t \notin[1, L]$, we have $p_{i}^{(t, I)}=p_{\text {synd }, i}^{(t, I)}=0$. The DE update equations for user 1 for $t \in[1, L]$ are given as (cf. [7])

$$
\begin{aligned}
& p_{1}^{(t, I+1)}=\epsilon_{\mathrm{s}_{1} \mathrm{~d}}\left(\frac{1}{w} \sum_{j=0}^{w-1} q_{1}^{(t+j, I)}\right)^{l_{1}-1}\left(\frac{1}{w} \sum_{j=0}^{w-1} q_{\mathrm{synd}, 1}^{(t+j, I)}\right)^{l_{\mathrm{synd}}^{1}} \\
& q_{1}^{(t, I+1)}=1-\left(1-\frac{1}{w} \sum_{k=0}^{w-1} p_{1}^{(t-k, I+1)}\right)^{r_{1}-1} \\
& p_{\mathrm{synd}, 1}^{(t, I+1)}=\epsilon_{\mathrm{s}_{1} \mathrm{~d}}\left(\frac{1}{w} \sum_{j=0}^{w-1} q_{1}^{(t+j, I)}\right)^{l_{1}}\left(\frac{1}{w} \sum_{j=0}^{w-1} q_{\mathrm{synd}, 1}^{(t+j, I)}\right)^{l_{\mathrm{synd}}^{1}-1} \\
& q_{\mathrm{synd}, 1}^{(t, I+1)}=1-\left(1-\frac{1}{w} \sum_{k=0}^{w-1} p_{\mathrm{synd}, 1}^{(t-k+1)}\right)^{r_{\mathrm{synd}}^{1}-1} \\
& \cdot\left(1-\frac{1}{w} \sum_{k=0}^{w-1} p_{\mathrm{synd}, 2}^{(t-k, I+1)}\right)^{r_{\mathrm{synd}}^{2}}
\end{aligned}
$$

The equations for user 2 are analogous. The coupling of the two users' codes in the decoding process manifests itself in the messages sent from the second layer check nodes (13).

Assuming symmetric channel conditions, both users can use codes $\mathcal{C}_{i}$ from the same ensemble $(l, r, L, w)$, and the relay can generate the syndrome bits using two codes $\mathcal{C}_{\text {synd }}^{i}$ from the same ensemble $\left(l_{\text {synd }}, r_{\text {synd }}, L, w\right)$. This means $l_{1}=l_{2}=l$, $r_{1}=r_{2}=r, l_{\text {synd }}^{1}=l_{\text {synd }}^{2}=l_{\text {synd }}$ and $r_{\text {synd }}^{1}=r_{\text {synd }}^{2}=r_{\text {synd }}$. The initial variable-to-check messages in the first iteration are equal for both users and both layers $p_{1}^{(t, 1)}=p_{\text {synd, } 1}^{(t, 1)}=p_{2}^{(t, 1)}=$ $p_{\text {synd }, 2}^{(t, 1)}=\epsilon_{\mathrm{sd}}$. With $r_{\text {synd }}=\frac{r}{2}$, (13) becomes equal to (12), i.e., the check-to-variable messages of the users in both layers are equal. This means that in the second iteration the variableto-check messages will be equal again, and via induction, the same will happen in all the following iterations. Due to the assumed symmetry, the equations for the second user are the same as for the first user. The DE for each user can therefore be written as

$$
\begin{aligned}
p_{i}^{(t, I+1)} & =\epsilon_{\mathrm{sd}}\left(\frac{1}{w} \sum_{j=0}^{w-1} q_{i}^{(t+j, I)}\right)^{l+l_{\mathrm{synd}}-1} \\
& =\epsilon_{\mathrm{sd}}\left(1-\frac{1}{w} \sum_{j=0}^{w-1}\left(1-\frac{1}{w} \sum_{k=0}^{w-1} p_{i}^{(t+j-k, I)}\right)^{r-1}\right)^{l+l_{\mathrm{synd}}-1}
\end{aligned}
$$


which is the update equation for a single-layer SC-LDPC code ensemble $\left(l+l_{\text {synd }}, r, L, w\right)$. The bilayer code ensembles for both users will therefore have the same DE thresholds as the single-layer ensemble.

Lemma 2. For symmetric channel conditions and $r_{\text {synd }}=$ $r / 2$, the design rate of the two-user bilayer code $\mathcal{C}_{\text {bl }}\left(l, l_{\text {synd }}, r, r_{\text {synd }}, L, w\right)$ for each source-destination link approaches that of the $\mathcal{C}\left(l+l_{\text {synd }}, r, L, w\right)$ single-layer ensemble as $w$ grows large.

Proof: The design rate of an SC-LDPC code is [7] $R=$ $1-\frac{N_{\mathrm{C}}}{N_{\mathrm{V}}}$, where $N_{\mathrm{V}}$ and $N_{\mathrm{C}}$ denote the number of variable nodes and check nodes, respectively, in the graph. The number of variable nodes per user is $N_{\mathrm{V}}=M L$. The number of checks per user in the first layer is

$$
N_{\mathrm{C}}=M \frac{l}{r}\left[L+1+w-2 \sum_{j=0}^{w-1}\left(\frac{j}{w}\right)^{r}\right]
$$

and the number of checks (which are shared by both users) in the second layer

$$
N_{\mathrm{C}, \text { synd }}=M \frac{2 l_{\text {synd }}}{r}\left[L+1+w-2 \sum_{j=0}^{w-1}\left(\frac{j}{w}\right)^{\frac{r}{2}}\right] .
$$

Since the checks in the second layer are shared equally between the two users, the effective number of checks per user is $N_{\mathrm{C} \text {,synd }}^{\text {eff }}=N_{\mathrm{C} \text {,synd }} / 2$ (cf. (6)). The rate of the bilayer code for each user is therefore $R_{\mathrm{bl}}=1-\frac{N_{\mathrm{C}}+N_{\mathrm{C}, \text { synd }} / 2}{M L}$. For large enough $w$, this can be approximated as

$$
R_{\mathrm{bl}} \approx 1-\frac{l+l_{\text {synd }}}{r}\left(1+\frac{w}{L}\right)
$$

which is the same expression as for the rate of an $(l+$ $\left.l_{\text {synd }}, r, L, w\right)$ code ensemble for large $w$.

Corollary 1. For symmetric channel conditions, the two-user bilayer $S C$-LDPC code $\mathcal{C}_{b l}\left(l, l_{\text {synd }}, r, r / 2, L, w\right)$ has the rate of the single-layer $\left(l+l_{\text {synd }}, r, L, w\right)$ code,

$$
\lim _{w \rightarrow \infty} \lim _{L \rightarrow \infty} R\left(l, l_{\text {synd }}, r, r / 2, L, w\right)=1-\frac{l+l_{\text {synd }}}{r},
$$

and for fixed $\left(l+l_{\text {synd }}\right) / r$, its BP threshold tends to the Shannon limit of that code,

$$
\lim _{\left(l+l_{\text {synd }}\right) \rightarrow \infty} \lim _{w \rightarrow \infty} \lim _{L \rightarrow \infty} \epsilon^{\mathrm{BP}}\left(l, l_{\text {synd }}, r, r / 2, L, w\right)=\frac{l+l_{\text {synd }}}{r} .
$$

Proof: From Lemmas 1 and 2 we know that in the limit of large $w$ and $L$, the bilayer code ensemble has the same rate and DE threshold as the single-layer $\left(l+l_{\text {synd }}, r, L, w\right)$ code ensemble. Therefore, the corollary follows from [7, Theorem 10 and Lemma 8].

Theorem 1. For a binary erasure relay channel with two sources, one relay, and one destination with symmetric channel conditions, there exists an SC-LDPC code $\mathcal{C}$ and an associated two-user bilayer code $\mathcal{C}_{b l}$ such that $\mathcal{C}$ achieves the capacity for both source-relay link and $\mathcal{C}_{b l}$ achieves capacity for both source-destination links. In addition, this code construction achieves the highest possible rate with DF relaying (cf. (2)).

Proof: The capacities of the source-relay links are $C_{\mathrm{sr}}=$ $1-\epsilon_{\mathrm{sr}}$. We use capacity-achieving SC-LDPC codes from the ensemble $\mathcal{C}(l, r, L, w)$, with

$$
\frac{l}{r}=\epsilon_{\mathrm{sr}}
$$

which are known to be asymptotically capacity achieving (cf. (4)), and therefore the relay will be able to decode successfully.

Let $N_{\mathrm{V}}$ be the number of variable nodes in $\mathcal{C}$. In the limit $L \rightarrow \infty$ there are $N_{\mathrm{C}}=\frac{l}{r} N_{\mathrm{V}}$ check nodes. The effective number of additional bits needed by the destination and provided by the relay is (cf. (6), (7), $\mu_{i}=1 / 2$ )

$$
N_{\mathrm{C}, \text { synd }}^{\mathrm{eff}}=N_{\mathrm{C}, \text { synd }} / 2=N_{\mathrm{V}}\left(C_{\mathrm{sr}}-C_{\mathrm{sd}}\right)=N_{\mathrm{V}}\left(\epsilon_{\mathrm{sd}}-\epsilon_{\mathrm{sr}}\right) .
$$

Remember that we chose $r_{\text {synd }}=r / 2$. The additional effective $N_{\mathrm{C}, \text { synd }}^{\text {eff }}$ check nodes from the second layer add $N_{\mathrm{C} \text {,synd }} r / 2=$ $r N_{\mathrm{C} \text {,synd }}^{\text {eff }}$ edges. The variable node degree $l_{\text {synd }}$ is

$$
l_{\text {synd }}=r N_{\mathrm{C}, \text { synd }}^{\mathrm{eff}} / N_{\mathrm{V}}=r\left(\epsilon_{\mathrm{sd}}-\epsilon_{\mathrm{sr}}\right) .
$$

From Corollary 1 together with (14) and (16) it follows that

$$
\lim _{w \rightarrow \infty} \lim _{L \rightarrow \infty} R\left(l, l_{\text {synd }}, r, r / 2, L, w\right)=1-\frac{l+l_{\text {synd }}}{r}=1-\epsilon_{\mathrm{sd}}
$$

and for fixed $\left(l+l_{\text {synd }}\right) / r$,

$$
\lim _{\left(l+l_{\text {synd }}\right) \rightarrow \infty} \lim _{w \rightarrow \infty} \lim _{L \rightarrow \infty} \epsilon^{\mathrm{BP}}\left(l, l_{\text {synd }}, r, r / 2, L, w\right)=\epsilon_{\mathrm{sd}},
$$

therefore we see that $\mathcal{C}_{\mathrm{bl}}$ achieves the capacity on the sourcedestination links $C_{\mathrm{sd}}=1-\epsilon_{\mathrm{sd}}$ for both users. The number of channel uses in the first two transmission phases is $n_{1}=$ $n_{2}=N_{\mathrm{V}}$. A capacity-achieving SC-LDPC code is used to transmit the $N_{\mathrm{C} \text {,synd }}$ syndrome bits in the third phase, using $n_{\mathrm{r}}=N_{\mathrm{C}, \text { synd }} / C_{\mathrm{rd}}$ channel uses. Therefore we have (with (15))

$$
\frac{n_{i}}{n_{1}+n_{2}+n_{\mathrm{r}}}=\frac{C_{\mathrm{rd}}}{2 C_{\mathrm{rd}}+2\left(C_{\mathrm{sr}}-C_{\mathrm{sd}}\right)}=\theta_{i}^{*}
$$

the optimum time allocation (1) which maximizes the achievable rate.

\section{NUMERICAL RESULTS}

The performance of a bilayer code can be assessed by comparing the DE thresholds of the first-layer code and of the overall bilayer code, respectively, to the Shannon limits [6]. Clearly, the overall system performance depends also on the code used on the relay-to-destination link, but that one is designed independently.

For the case considered, there are two first-layer codes and two bilayer codes in the system, one for each user. Tables I and II show a number of two-user bilayer SC-LDPC code ensembles and their thresholds. For all results, $L=100$. In Table I, the same code ensemble was used for the first layer of both users, $\mathcal{C}_{i}(3,10,100, w)$, which is a design for equal EP on both source-relay links. The first row in the table shows the symmetric case. We have confirmed that the DE threshold 
TABLE I

DE THRESHOLDS OF SOME TWO-USER SC-LDPC BILAYER CODES FOR THE CASE OF SYMMETRIC SOURCE-RELAY LINKS.

\begin{tabular}{cccccccccccccccc}
\hline$\left(l_{\text {synd }}^{1}, r_{\text {synd }}^{1}\right)$ & $\left(l_{\text {synd }}^{2}, r_{\text {synd }}^{2}\right)$ & $w$ & $\mu$ & $R_{\mathrm{bl}}^{1}$ & $\epsilon_{\mathrm{s}_{1} \mathrm{~d}}^{\mathrm{Sh}}$ & $\epsilon_{\mathrm{s}_{1} \mathrm{~d}}^{\mathrm{BP}}$ & $\mathrm{Gap}$ & $R_{\mathrm{bl}}^{2}$ & $\epsilon_{\mathrm{s}_{2} \mathrm{~d}}^{\mathrm{Sh}}$ & $\epsilon_{\mathrm{s}_{2} \mathrm{~d}}^{\mathrm{BP}}$ & $\mathrm{Gap}$ & $R_{\text {sum }}$ \\
\hline$(2,5)$ & $(2,5)$ & 3 & 1 & 0.4907 & 0.5093 & 0.4989 & 0.0104 & 0.4907 & 0.5093 & 0.4989 & 0.0104 & 0.7417 \\
$(2,5)$ & $(4,10)$ & 3 & 0.5 & 0.5585 & 0.4415 & 0.4268 & 0.0147 & 0.4222 & 0.5778 & 0.5631 & 0.0147 & 0.7488 \\
$(2,5)$ & $(6,15)$ & 3 & 0.33 & 0.5924 & 0.4076 & 0.3792 & 0.0284 & 0.3882 & 0.6117 & 0.5835 & 0.0282 & 0.7524 \\
$(2,5)$ & $(8,20)$ & 3 & 0.25 & 0.6128 & 0.3872 & 0.3357 & 0.0515 & 0.3678 & 0.6321 & 0.5807 & 0.0514 & 0.7546 \\
$(2,5)$ & $(8,20)$ & 4 & 0.25 & 0.6094 & 0.3906 & 0.3653 & 0.0253 & 0.3618 & 0.6382 & 0.6129 & 0.0253 & 0.7535 \\
\hline
\end{tabular}

TABLE II

DE THRESHOLDS OF SOME TWO-USER SC-LDPC BILAYER CODES FOR THE CASE OF ASYMMETRIC SOURCE-RELAY LINKS.

\begin{tabular}{|c|c|c|c|c|c|c|c|c|c|c|c|c|}
\hline$\left(l_{\text {synd }}^{1}, r_{\text {synd }}^{1}\right)$ & $\left(l_{\text {synd }}^{2}, r_{\text {synd }}^{2}\right)$ & $w$ & $\mu$ & $R_{\mathrm{bl}}^{1}$ & $\epsilon_{\mathrm{s}_{1} \mathrm{~d}}^{\mathrm{Sh}}$ & $\epsilon_{\mathrm{s}_{1} \mathrm{~d}}^{\mathrm{BP}}$ & Gap & $R_{\mathrm{bl}}^{2}$ & $\epsilon_{\mathrm{s}_{2} \mathrm{~d}}^{\mathrm{Sh}}$ & $\epsilon_{\mathrm{s}_{2} \mathrm{~d}}^{\mathrm{BP}}$ & Gap & $R_{\text {sum }}$ \\
\hline$(2,5)$ & $(3,10)$ & 4 & 1 & 0.6574 & 0.3426 & 0.3157 & 0.0269 & 0.3823 & 0.6177 & 0.5908 & 0.0269 & 0.8616 \\
\hline$(2,5)$ & $(6,20)$ & 8 & 0.5 & 0.7021 & 0.2979 & 0.2646 & 0.0333 & 0.3161 & 0.6839 & 0.6506 & 0.0333 & 0.8717 \\
\hline
\end{tabular}

obtained in this case is the same as for the single-layer $(l+$ $\left.l_{\text {synd }}=5,10,100, w\right)$ ensemble, as predicted by Lemma 1 . The next rows show codes for increasingly different EP on the source-destination links. This is achieved by increasing the node degrees of the $\mathcal{C}_{\text {synd }}^{2}$ ensemble while keeping $\mathcal{C}_{\text {synd }}^{1}$ fixed, thereby changing the ratio $\mu$ of effective syndrome bits generated for each source. The first four rows in the table use $w=3$. This gives the design rate of the first layer codes $R_{i}=0.6941$, a Shannon limit of $\epsilon^{\mathrm{Sh}}=0.3058$, and a DE threshold of $\epsilon^{\mathrm{BP}}=0.2865$, which corresponds to a gap of 0.0193. The gaps of the bilayer codes to the Shannon limit are very low, starting with 0.0104 for the symmetric case, increasing slightly with stronger asymmetry, up to 0.0514 for $\mu=0.25$. It is shown in the fifth row of the table that the gap can be reduced to 0.0253 by using $w=4$. For the firstlayer code we have then $R_{i}=0.6914$, a Shannon limit of $\epsilon^{\mathrm{Sh}}=0.3086$, and a DE threshold of $\epsilon^{\mathrm{BP}}=0.2865$, which corresponds to a gap of 0.0221 .

Table II shows DE results for the case of asymmetric source-relay and source-destination links. Source one uses $\mathcal{C}_{1}(4,20,100, w)$ and source two $\mathcal{C}_{2}(8,20,100, w)$. For the first row, $w=4$, which leads to $R_{1}=0.7941, R_{2}=0.5881$. The gaps to the Shannon limit have been found to be 0.0089 and 0.0142 , respectively. The second row uses $w=8$, where we have $R_{1}=0.7863, R_{2}=0.5726$, and gaps to the Shannon limit of 0.0166 and 0.0275 , respectively. The bilayer codes exhibit gaps to capacity that are again very low (0.0269 and 0.0333). In the light of this good performance, we conjecture that our design has the potential to achieve capacity also in the case of asymmetric channel conditions.

The tables also state the system sum rate, assuming a capacity of the relay-to-destination link of $C_{\mathrm{rd}}=0.8$. The sum rate is given by

$$
R_{\text {sum }}=2 \frac{R_{\mathrm{r}} R_{1}}{R_{\mathrm{r}}\left(1+R_{1} / R_{2}\right)+2 R_{1}-R_{\mathrm{bl}}^{1}-R_{\mathrm{bl}}^{2} R_{1} / R_{2}} .
$$

\section{CONCLUSIONS}

In this paper, we presented two-user bilayer SC-LDPC codes for the time-division multiple access relay channel. We proved that the coding scheme achieves the maximum possible decode-and-forward rate for the TDMA relay channel with symmetric binary erasure channel links. Capacity approaching performance was demonstrated for asymmetric scenarios using density evolution. Future extensions of this work include the proof that capacity can be achieved in scenarios with asymmetric channel conditions and consideration of more general channel models like AWGN and fading channels.

\section{REFERENCES}

[1] E. C. van der Meulen, "Three-terminal communication channels," Advances in Applied Probability, vol. 3, no. 1, pp. 120-154, 1971.

[2] T. Cover and A. El Gamal, "Capacity theorems for the relay channel," IEEE Trans. Inform. Theory, vol. 25, no. 5, pp. 572-583, Sept. 1979.

[3] A. Stefanov and E. Erkip, "Cooperative coding for wireless networks," IEEE Trans. Commun., vol. 52, no. 9, pp. 1470-1476, Sep. 2004.

[4] B. Zhao and M. Valenti, "Distributed turbo coded diversity for relay channel," IEEE Power Electron. Lett., vol. 39, no. 10, pp. 786 - 787, May 2003.

[5] A. Chakrabarti, A. de Baynast, and A. Sabharwal, "Low density parity check codes for the relay channel," IEEE J. Sel. Areas Commun., Jan. 2007.

[6] P. Razaghi and W. Yu, "Bilayer low-density parity-check codes for decode-and-forward in relay channels," IEEE Trans. Inf. Theory, vol. 53, no. 10, pp. 3723-3739, 2007.

[7] S. Kudekar, T. Richardson, and R. Urbanke, "Threshold saturation via spatial coupling: Why convolutional LDPC ensembles perform so well over the BEC," IEEE Trans. Inf. Theory, vol. 57, no. 2, pp. 803-834, Feb. 2011

[8] Z. Si, R. Thobaben, and M. Skoglund, "Bilayer LDPC convolutional codes for half-duplex relay channels," in Proc. IEEE Int. Symp. Inf. Theory, Aug. 2011, pp. 1464-1468.

[9] G. Kramer and A. van Wijngaarden, "On the white Gaussian multipleaccess relay channel," in Proc. IEEE Int. Symp. Inf. Theory, Jun. 2000, p. 40 .

[10] C. Hausl, "Joint network-channel coding for wireless relay networks," Ph.D. dissertation, TU München, 2008.

[11] R. Ahlswede, N. Cai, S. Li, and R. Yeung, "Network information flow," IEEE Trans. Inf. Theory, vol. 46, no. 4, pp. 1204-1216, Jul. 2000.

[12] C. Hausl, F. Schreckenbach, I. Oikonomidis, and G. Bauch, "Iterative network and channel decoding on a tanner graph," in Proc. Allerton Conf. on Commun., Control and Computing, 2005.

[13] J. Li, J. Yuan, R. Malaney, M. Azmi, and M. Xiao, "Network coded LDPC code design for a multi-source relaying system," IEEE Trans. Wireless Commun., vol. 10, no. 5, pp. 1538-1551, May 2011.

[14] R. Youssef and A. Graell i Amat, "Distributed Serially Concatenated Codes for Multi-Source Cooperative Relay Networks," IEEE Trans. Wireless Commun., vol. 10, no. 1, pp. 253-263, 2011. 